\title{
Article
}

\section{Formation of the Optimal Load of High School Students Using a Genetic Algorithm and a Neural Network}

\author{
Elena Petrovna Dogadina ${ }^{1, *}$, Michael Viktorovich Smirnov ${ }^{1}$, Aleksey Viktorovich Osipov ${ }^{1}$ (i) \\ and Stanislav Vadimovich Suvorov ${ }^{2}$ \\ 1 Department of Data Analysis and Machine Learning, Federal State Budgetary Institution of Higher \\ Education, Financial University under the Government of the Russian Federation, 105187 Moscow, Russia; \\ MVSmirnov@fa.ru (M.V.S.); AVOsipov@fa.ru (A.V.O.) \\ 2 Federal State Autonomous Educational Institution of Higher Education, Moscow Polytechnic University, \\ 107023 Moscow, Russia; ssw1168@mail.ru \\ * Correspondence: delena09@yandex.ru; Tel.: +7-915-791-8524
}

check for updates

Citation: Dogadina, E.P.; Smirnov, M.V.; Osipov, A.V.; Suvorov, S.V. Formation of the Optimal Load of High School Students Using a Genetic Algorithm and a Neural Network. Appl. Sci. 2021, 11, 5263. https:// doi.org/10.3390/app11115263

Academic Editor: Giancarlo Mauri

Received: 18 May 2021

Accepted: 3 June 2021

Published: 6 June 2021

Publisher's Note: MDPI stays neutral with regard to jurisdictional claims in published maps and institutional affiliations.

Copyright: (c) 2021 by the authors. Licensee MDPI, Basel, Switzerland. This article is an open access article distributed under the terms and conditions of the Creative Commons Attribution (CC BY) license (https:// creativecommons.org/licenses/by/ $4.0 /)$.

\begin{abstract}
The problem of the effectiveness of teaching can be successfully solved only if the high quality of lessons is supported by well-organized homework of students. The question of homework occupies one of the main places in educational activities since this question is directly related to the health of the child. A competent approach to minimizing the time for completing homework, taking into account the maximum efficiency obtained from the learning process, can preserve the health of students to some extent. The article describes a method for obtaining the most comfortable results of the process of completing homework, which are a Pareto set. This method is implemented using a genetic algorithm and queuing theory, and the selection of homework is carried out on the basis of intellectual analysis of the text of tasks and is a scale of a certain range. The proposed algorithm successfully obtains the solutions of the Pareto set and minimizes the efforts of school students while achieving the maximum efficiency of the educational process to preserve their health. Compared with other known algorithms, the results obtained show that the proposed algorithm demonstrates fairly accurate optimization characteristics presented in the form of a Pareto set. Furthermore, combining a genetic algorithm, queuing theory apparatus, and a neural network makes it possible to model the studied subject area more accurately.
\end{abstract}

Keywords: neural networks; genetic algorithm; queuing theory; education

\section{Introduction}

Modern schoolchildren have a heavy training load. The experts are alarmed as physical fatigue and mental exhaustion of schoolchildren have negative consequences for their health. According to the Federal Consumer Protection Oversight Service, the number of entirely healthy children in Russia does not exceed $12 \%$. More than half of schoolchildren aged $7-9$ and $60 \%$ of high school students are diagnosed with chronic illness. Schools apply various health-saving technologies but at the same time strive to use the maximum permitted number of academic hours to complete a complex educational program.

The Federal Consumer Protection Oversight Service developed the SanPiN "Requirements for the conditions and organization of training in educational institutions" [1] concerning the health and safety criteria regarding the conditions and organization of education of schoolchildren. Here are some figures: the maximum study time per week ranges from 21 academic hours (6-7-year-old children) to 34 academic hours (16-17-yearolds); the maximum homework time ranges from $1.5 \mathrm{~h}$ per day (7-9-year-olds) to $3.5 \mathrm{~h}$ (15-17-year-olds). To give a child a good education without harm to his or her health and psyche, it is necessary to understand what kind of study load will be optimal. The authors propose to minimize the efforts of schoolchildren while achieving maximum efficiency of the educational process. 
To successfully solve the problem of multi-purpose optimization with limitations, the authors proposed a genetic algorithm in this article, the chromosome matching function of which is defined for each individual (part of a chromosome) and represents the value of the target function, calculated by vector package, depending on the criteria. The authors used the apparatus of queuing theory to obtain optimality criteria. Given the relevance of the multifactor optimization problem, various approaches to its solution have found their application in a large number of scientific studies and are reflected in many publications. There are many works from various subject areas, the core of which is the use of a genetic algorithm [2-15]. In the analyzed publications, the authors try to adapt this algorithm to the peculiarities of a specific optimization problem. For example, Kamal, Butt, and Anjum [2] used a two-point mixing technique in which two random chromosomes of the previous generation were selected as parents. The definition of a soft gene has been introduced as a gene that can contain more than one possible value. Viana, Junior, and Contreras [3] improved the standard mixing and mutation operators to avoid premature convergence. This result was achieved thanks to the mixing operator developed by the authors, which is based on a random choice. Rarità et al. [4] developed a mechanism for choosing an initial population, which provides a high convergence rate of the genetic algorithm.

For the successful solution of multicriteria optimization problems, researchers in recent years have applied various types of evolutionary algorithms (EA), particle swarm optimization (PSO), random search with backtracking (BSA), and hybrid algorithms [5,12-15]. Y. Hu et al. [5] presented a hybrid algorithm based on GA-PSO-BPNN (genetic algorithmparticle swarm optimization-neural network training), combining the advantages of GA local search and PSO global search to eliminate the disadvantage in which BPNN easily hits the local optimum. The authors of the article [14] proposed a hybrid algorithm that successfully combines the features of conventional DE and PSO algorithms and overcomes their drawbacks. Hybridization was performed by injecting the DE/rand/1 and $\mathrm{DE} /$ current-to-best/ 1 mutation operators from the DE algorithm into the PSO algorithm rate update equation, with the adaptive normalized interval between populations used to select the appropriate mutation operator for the current optimization condition. The authors of the article [15] compared their proposed ABSA method with three classical BSA, GA, and DE in 15 test functions. They noted that in terms of the minimum consumption of computer resources, their new method is comparable to GA and DE, and in terms of operating time, it is 9 to 6 ahead of the rest.

The choice of a genetic algorithm as an optimization method is associated with the consideration of the following main criteria: the required accuracy of the sought solution, the search speed for the optimization method, the minimum computer resource consumption (lower minimum total cost), as well as the implementation time of the method. In addition, the choice of this optimization method depends on such features of mathematical models of problems as the dimension of the modeled system and the presence of discrete components in the vector of the optimized parameters.

To increase the optimization performance, especially when solving complex multipurpose optimization problems, the authors proposed a solution that combines the principles of a genetic algorithm, a neural network, and a non-stationary queuing system. This allows for varying the system's parameters and having an unlimited dimension of the model, as well as the optimized parameters themselves can be represented both in the form of a scalar and a vector.

To summarize, we can say that the main contribution to this document can be described as follows:

- This article formulates a methodology for multicriteria optimization of the learning process for schoolchildren with the ability to use a large number of target functions and a high dimension of the system.

- The authors of this article propose to combine a neural network, a system functioning model based on the queuing theory apparatus, and a genetic algorithm, so that, on the one hand, it would be possible to build a model of the dependence of the effectiveness 
of tasks for independent work of students on the types of activities, subject of the task, etc., and on the other hand, use this model to select the optimal parameters of the system (for example, the time to complete the task).

- To improve the optimization performance, especially when solving multicriteria optimization problems with a high dimension of the system, a genetic algorithm was proposed, some of the initial data of which were obtained using a neural network and a service system. Optimization results are Pareto set.

\section{Research of the Subject Area}

To assimilate the material effectively and at the same time to minimize the load on schoolchildren, it is necessary to design homework so that, on the one hand, the assignments are practical; on the other hand, they are not extraordinarily complex and overburdened.

Any training course shall consist of topics that together determine the content of the course. The homework assignments may be on the same topic, or they may refer to several topics so that the student has the opportunity to repeat the material, and the opportunity to look at the material passed under a different perspective. Furthermore, if the assignments involve different parts of the learner's brain, and different types of memory, it also contributes to more effective learning. In this connection, teachers often choose exercises for the assignments in such a way that the students can draw something, say something, solve thematic crosswords, compose poems, write lyrics, etc. That is, if the assignment includes different types of activities, it will be more efficient in terms of assimilation. However, if the task is overloaded, the schoolchild will become more tired, which will affect efficiency.

Thus, we have an optimization problem in which the activities of the schoolchild and the subject of the assignment should be chosen in such a way that, on the one hand, maximum efficiency in learning is achieved and, on the other hand, the time burden is reduced. This optimization problem is not trivial because the identification of the regularities between activities, the subject of the assignments, and, after all, the learner's abilities and efficiency, is a problem related to physiology, psychology, pedagogy, and other disciplines. In this case, neural networks are the most appropriate tool for detecting these hidden dependencies $[16,17]$.

The authors of this article propose to combine the neural network and genetic algorithms to construct a model of the dependency of the effectiveness of assignments on activities, topics, time, and other features, so that it would be possible to use this model to find the optimal parameters of the assignment.

The authors have identified the following numerical characteristics of assignments for the school students:

- Number of topics the assignment deals with;

- Number of types of activities students use while doing the assignment;

- Number of questions in the assignment;

- Length of the assignment;

- Complexity in formulating the assignment;

- Age of a student;

- Sex of a student.

The composition of the selected characteristics may change. The number of topics covered by the assignment - this value is determined by lexemes specific to a particular topic. For example, the "electric current" lexeme means that the assignment has a topic related to electricity. The length of the assignment is the number of words required to formulate the assignment. The complexity of the assignment formulation is an expert value determined in scores in the range from 1 (the assignment is formulated clear and unambiguously) to 3 (there are redundant data and ambiguous formulations). The age of the school student is in years. Sex is a binary value. The target value will be a number that ranges from 0 to 1 , where 0 is an entirely useless assignment with no impact on the 
perception of the material and 1 is an ideal to be pursued (i.e., an assignment that allows the school student to learn the material thoroughly).

We used an MLP (Multilayer Perceptron) neural network trained by the Resilient Propagation (RProp) method to build the model. We implemented the neural network using the Neural Excel VBA Extension Pack Software [18]. The main advantage of this method is the simplicity of implementation at a high convergence rate and low requirements for the error in calculating the gradient. The algorithm uses the so-called "learning by epochs" when the weights are corrected after all the training samples are presented to the network.

To train the neural network, we prepared a training set of 250 tasks, for each of which the efficiency values were derived by the method of expert evaluation. For testing, we have prepared a test sample of 50 items. Empirically, we have established the neural network structure that gives the maximum forecasting accuracy (Figure 1). It contains the following parameters:

- Input layer-7 neurons;

- Hidden layer-19 neurons;

- Output layer-1 neuron.

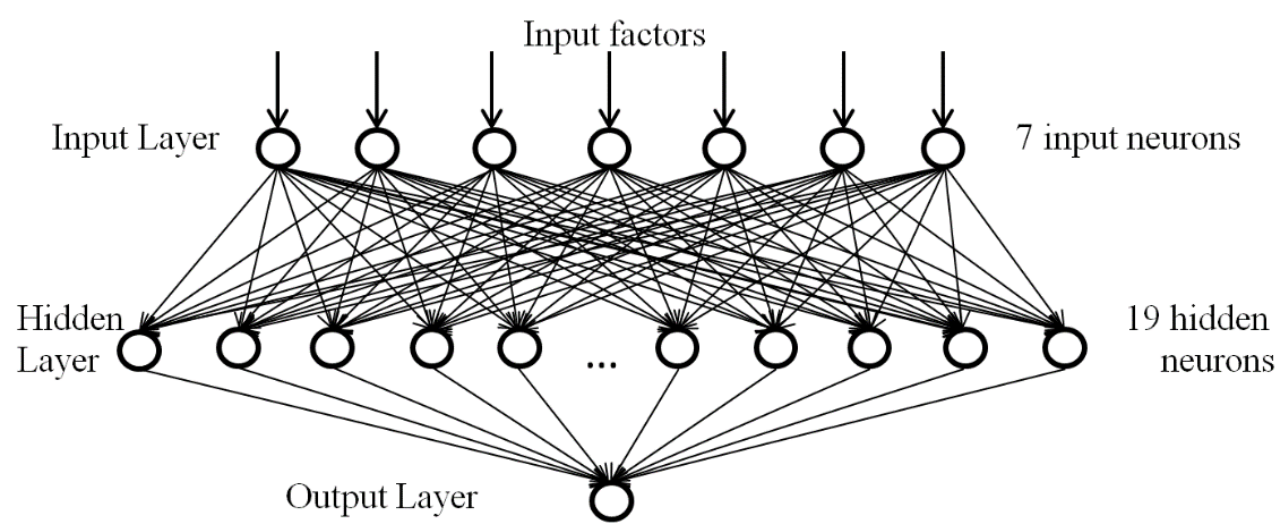

Figure 1. The neural network structure.

The model predicted the effectiveness of the assignments with $87 \%$ accuracy on the training set and $82 \%$ accuracy on the test set.

\section{Materials and Methods}

The article describes a mathematical model of multicriteria optimization of homework fulfillment by students based on the application of queuing theory, neural networks, and stochastic optimization methods.

The choice of a genetic algorithm as an optimization method is associated with research, during which the genetic algorithm has proven itself to be the best. Methods of random search with a return on an unsuccessful step and the ant colony method were considered as analogs. Comparison of the effectiveness of the application of optimization methods was carried out according to these criteria: 1 -ease of use; 2 - the minimum time spent on implementation; 3-the accuracy of the result; 4-the possibility of rapid modernization of the method; 5-search speed for the optimization method; 6-the minimum cost of computer resources (lower minimum total cost).

The considered structure of organizing homework can be represented on the basis of non-stationary multiphase single-channel and multichannel queuing systems with waiting and with failure [19]. To describe the functioning of the process of receipt and service of claims in the system, we will use the following main characteristics: the capacity of the source of applications $N_{0}(t)$, the length of the queue of applications $Q_{0}(t)$ and the number of service devices $M_{0}(t)$ [19].

At any given time, the production process can be in one of the states defined by characteristics such as the number of service devices in operation $M(t)$, the number of 
devices in use $Z(t)$ and the number of requests in the queue $Q(t)$. Let $S(m, z, q)$ represent the condition when the system uses $m$ devices, they employ the service of devices $z$, and $q$ applications is in the queue. To describe the intensity of applications in the system and the intensity of service applications, we introduce vector quantities $\lambda(t)$ and $\mu(t)$. In the course of statistical analysis, the values $\lambda(t)$ and $\mu(t)$ are subject to the exponential distribution law. Verification of these hypotheses about the exponential distribution law was carried out on the basis of the criterion $\chi^{2} K$. Pearson.

A mathematical model has been developed for the functioning of processes based on the construction of systems of Kolmogorov differential equations on the considered time interval $t \in\{0, T\}[9,11,19,20]$. The model has such form:

$$
\frac{d p_{a}(t)}{d t}=-\sum_{\substack{a, b \in N^{\prime} \\ b \neq a}} d_{a b}(t) \cdot p_{a}(t)+\sum_{\substack{a, c \in N^{\prime} \\ c \neq a}} d_{c a}(t) \cdot p_{c}(t)
$$

where $N^{\prime}$-a plurality of pairs of indexes of the states $N^{\prime}=\left\{(a, b) \in N^{2} \mid d_{a b}=\left(S_{a}, S_{b}\right)\right\} \mathrm{N}$ $N=\{0,1,2, \ldots\}$.

The use of variables $a, b, c$ in the mathematical model suggests that the system is in states $S\left(m_{a}, z_{a}, q_{a}\right), S\left(m_{b}, z_{b}, q_{b}\right)$, and $S\left(m_{c}, z_{c}, q_{c}\right)$ respectively.

The initial state is expressed as:

$$
p_{a}(0)=p_{a 0}>0, \quad a \in N
$$

It is also necessary to observe the condition of normalization:

$$
\sum_{a \in N} p_{a}(t)=1
$$
follows:

The density $d_{a b}(a \neq b)$ of the transition from the $S_{a}$ state to the $S_{b}$ state is defined as

$$
d_{a b}=\left\{\begin{array}{c}
\mu_{a}(t), b=a-1, t \in[0, T] \\
\lambda_{a}(t), b=a+1, t \in[0, T] \\
0, a \neq b, a=0,1,2 \ldots
\end{array}\right.
$$

We represent the process of optimizing the time for completing homework for students on the basis of a genetic algorithm [3-6] and a multicriteria control problem [19,20].

Let us introduce the term chromosome, which represents each value of the vector of the optimized parameters $X=\left(X_{1}, X_{2}, \ldots, X_{n}\right) \in \Omega_{\partial o}$.

1. The values of the vector of the optimized parameters are presented as chromosomes $X=\left(X_{1}, X_{2}, \ldots, X_{n}\right) \in \Omega_{\partial o}$. Moreover, each individual of the population must belong to the range of permissible values $\Omega_{\partial \circ}$. An individual $X_{i, r} i=1 . . N_{-} p o p$ is understood as the corresponding value of the vector $X=\left(X_{i, 1}, X_{i, 2}, \ldots, X_{i, n}\right) \in \Omega_{\partial o}$, in which the number of changes in individuals and the gene depends on the number of generations $N_{\text {pok }}$.

2. The chromosome matching function is determined for each individual separately and represents the value of the objective function, calculated using vector convolution, depending on the criteria.

3. The selected genes (components of an individual $\left(X_{i, 1}, X_{i, 2}, \ldots, X_{i, n}\right)$ ) for crossing must not coincide, and the value of these genes must be within the range of acceptable values, otherwise, the choice of other genes.

4. To carry out a mutation, it is necessary to select a gene that undergoes mutation and designate its serial number in the population; a bit in a gene that needs to be mutated.

5. As a condition for the completion of the process, we use the achievement of a given number of generations. 
On the basis of an algorithm based on a genetic algorithm, it is required to determine the Pareto set of the required optimization parameter. By the Pareto set, we mean the set of unconditionally incomparable alternatives that remain after discarding all unconditionally worse alternatives.

Let us reduce the problem of determining the optimal time for completing homework by students with several optimization criteria to the following form:

$$
\begin{gathered}
K=f(p(t), X) \rightarrow \min (\max ) \\
K=\left(K_{1}, K_{2}, \ldots, K_{r}\right), \\
\psi_{i}(p(t), X) \leq 0, \quad i=\overline{1, l} \\
X=(\lambda, \mu, M, Q) \in \Omega_{d o p} \\
\frac{d p(t)}{d t}=f(X(t), p(t)) \\
p_{a}(0)=p_{a 0}, a \in N \\
\sum_{a \in N} p_{a}(t)=1
\end{gathered}
$$

where $K$-vector-function of the selected criteria of optimality of production processes, $X$-vector of optimized parameters of the system, which depend on the probability density of transitions of the system, $\lambda$-vector of intensities of input flows of applications, $\mu-$ vector of intensities of their service, $M$-the number of service devices, $Q$-the length of the system queue, $p(t)$-vector-function of probabilities of the states of the system in the considered time interval $t \in\{0, T\}$, determined by the model of the form (7-9). The system of constraints (5) and the expression (6) define the scope of acceptable solutions to the problem.

The developed algorithm assumes the possibility of solving the problem of multicriteria control of the homework processes by students with the maximum possible efficiency of tasks and minimum labor costs for their implementation:

$$
\begin{gathered}
F(\xi, K(X)), \\
X=\left(X_{1}, X_{2}, \ldots, X_{n}\right) \in \Omega_{\text {dop }},
\end{gathered}
$$

where $\xi=\left(\xi_{1}, \xi_{2}, \ldots, \xi_{n}\right)$-weights of the relative importance of the criteria.

In this paper, the weight coefficients of the relative importance of the criteria are given in accordance with the conditions:

$$
\begin{gathered}
0 \leq \xi_{j} \leq 1, j=1 \ldots n \\
\sum_{j=1}^{n} \xi_{j}=1, j=1 \ldots n
\end{gathered}
$$

\section{Results}

We will describe the work on finding the optimal time for students to complete their homework using a non-stationary multichannel queuing system with a limited waiting queue.

In this optimization problem, we represent students of 6 classes doing their homework as service channels; applications are tasks in subjects; the input stream is formed by the teachers' requirements for students to complete their homework in accordance with SanPiN and Federal State Educational Standard (for example, the full employment of the student during the time allotted for homework).

It is necessary to introduce the following designations: $M$-the number of students (the model takes into account the possibilities of performing both individual tasks and project tasks for a group of students); $Q$ - the length of the queue of items with tasks; $\lambda(t)$ - the intensity of receipt of a set of tasks in subjects, distributed according to the 
exponential distribution law; $\mu(t)$ — the intensity of the student's homework, distributed according to the exponential distribution law; $T$-the considered operating time of the system, limited to a $2.5 \mathrm{~h}$ working day (for students of 6 grades).

Since the software implementation of the optimization problem posed is solved in the MATLAB environment (in which the variable indices start from one, and not from zero), the mathematical model of the functioning of the homework process by students has the form (10-12). Let $p_{k}$ the probability of the system being in a state in which it is necessary to complete tasks in $k$ subjects, $k=\overline{2, M+Q+1}$.

$$
\begin{aligned}
& \frac{d p_{1}(t)}{d t}=-\lambda p_{1}(t)+\mu p_{2}(t) \\
& \frac{d p_{i}(t)}{d t}=-(\lambda+(i-1) * \mu) p_{i}(t)+\lambda p_{i-1}(t)+i * \mu p_{i+1}(t), i=\overline{2, M} ; \\
& \frac{d p_{j+M}(t)}{d t}=-(\lambda+M * \mu) p_{j+M}(t)+\lambda p_{j+M-1}(t)+M * \mu p_{j+M+1}(t), j=\overline{1, Q} ; \\
& \frac{d p_{Q+M+1}(t)}{d t}=\lambda p_{Q+M}(t)-M * \mu p_{Q+M+1}(t) ; \\
& \quad p_{k}(0)=p_{k 0}>0, \quad k=\overline{1, M+Q+1}
\end{aligned}
$$

In addition, the normalization condition must be met.

$$
\sum_{k} p_{k}(t)=1, t \in[0, T]
$$

The parameter to be optimized is the average time for completing homework by one student, which is determined through the intensity and is equal to the reciprocal of the average time for making $\bar{t}_{\text {lesson }}$ homework $\mu=\frac{1}{\bar{t}_{\text {lesson }}}$.

We will use the following characteristics as optimality criteria:

1. The average relative time for completing homework, taking into account the difficulty of a particular subject, is as follows:

$$
K_{1}=\frac{1}{M \times T} \times \bar{t}_{\text {lesson }} \rightarrow \min
$$

where $\bar{t}_{\text {lesson }}$-average time to complete homework (min.) for one lesson.

The time limit for completing homework (min.) for one lesson is as follows:

$$
t_{\text {lesson }}=60 \times D \frac{B_{i}}{\sum_{i} B_{i} P_{i}}
$$

where

$D$-the time limit for completing homework during the week, according to hygiene requirements (hours);

$B_{i}$ - the difficulty of a separate $i$ subject;

$P_{i}$ - the number of hours in the curriculum for the $i$ subject.

2. Average relative efficiency of homework in terms of material assimilation:

$$
\begin{gathered}
K_{2}=\frac{\overline{E f} \times \bar{t}_{\text {lesson }}}{E f_{\max }} \rightarrow \text { max }, \\
\overline{E f}=a_{N} * k o l,
\end{gathered}
$$

where $\bar{t}_{\text {lesson }}$ is the average time for completing homework (min.) for one lesson; $\overline{E f}$ - the average efficiency of assignments in subjects assigned to the home; $E f_{\max }-$ maximum efficiency, $a_{N}$-task efficiency coefficient obtained using a neural network, kol-number of tasks. 
It is necessary to impose the condition on the parameter to be optimized with a constant average time for completing homework:

$$
a \leq \mu(t)=\mu \leq b,
$$

where $a$ and $b$ are determined by the decision-maker.

So, the problem of multicriteria optimization is formulated as follows: determine the optimal value at which the average relative time is for completing homework, taking into account the difficulty of an individual subject (13); when it reaches a minimum and the average relative efficiency of the task (14) reaches a maximum when conditions (10-12) and (15).

Here is an example of how one child can optimize individual homework. We have the following data: $M=1$-the number of students; $Q=5$-the length of the queue of subjects for which homework is given; $\lambda=1 / 15 \mathrm{~min}^{-1}$-the intensity of the input flow of incoming applications; $T=2.5 \mathrm{~h}$. The accuracy of solving the system of equations (10-12) by the Runge-Kutta method is $4-5$ orders of magnitude of accuracy $\Delta t=0.1 \mathrm{~min}$; $\mu_{i} \in[0.01 ; 1] \mathrm{min}^{-1}, i=\overline{1, n}$.

The solution of this problem by the method using the genetic algorithm is presented in Table 1, and the corresponding Pareto-optimal set is shown in Figure 2. The graph of the probability distribution of the system being in a certain state is shown in Figure 3. In this example, we assume the size of the population $N_{-} p o p=25$ and the number of generations N_pok $=20$.

Table 1. Optimization results by the method using the genetic algorithm.

\begin{tabular}{cccc}
\hline & $\boldsymbol{K}_{1}$ & $\boldsymbol{K}_{2}$ & $\boldsymbol{\mu}_{\mathbf{~ m i n}}^{-\mathbf{1}}$ \\
\hline 0.0 & 0.0682 & 0.0943 & 0.9869 \\
0.1 & 0.0688 & 0.0951 & 0.9721 \\
0.2 & 0.0670 & 0.0927 & 0.9867 \\
0.3 & 0.0680 & 0.0941 & 0.9731 \\
0.4 & 0.0676 & 0.0934 & 0.9798 \\
0.5 & 0.0679 & 0.0939 & 0.9749 \\
0.6 & 0.2570 & 0.3661 & 0.25 \\
0.7 & 0.4869 & 0.7573 & 0.1209 \\
0.8 & 0.4544 & 0.6933 & 0.1477 \\
1.0 & 0.7296 & 1.5957 & 0.0574 \\
\hline
\end{tabular}




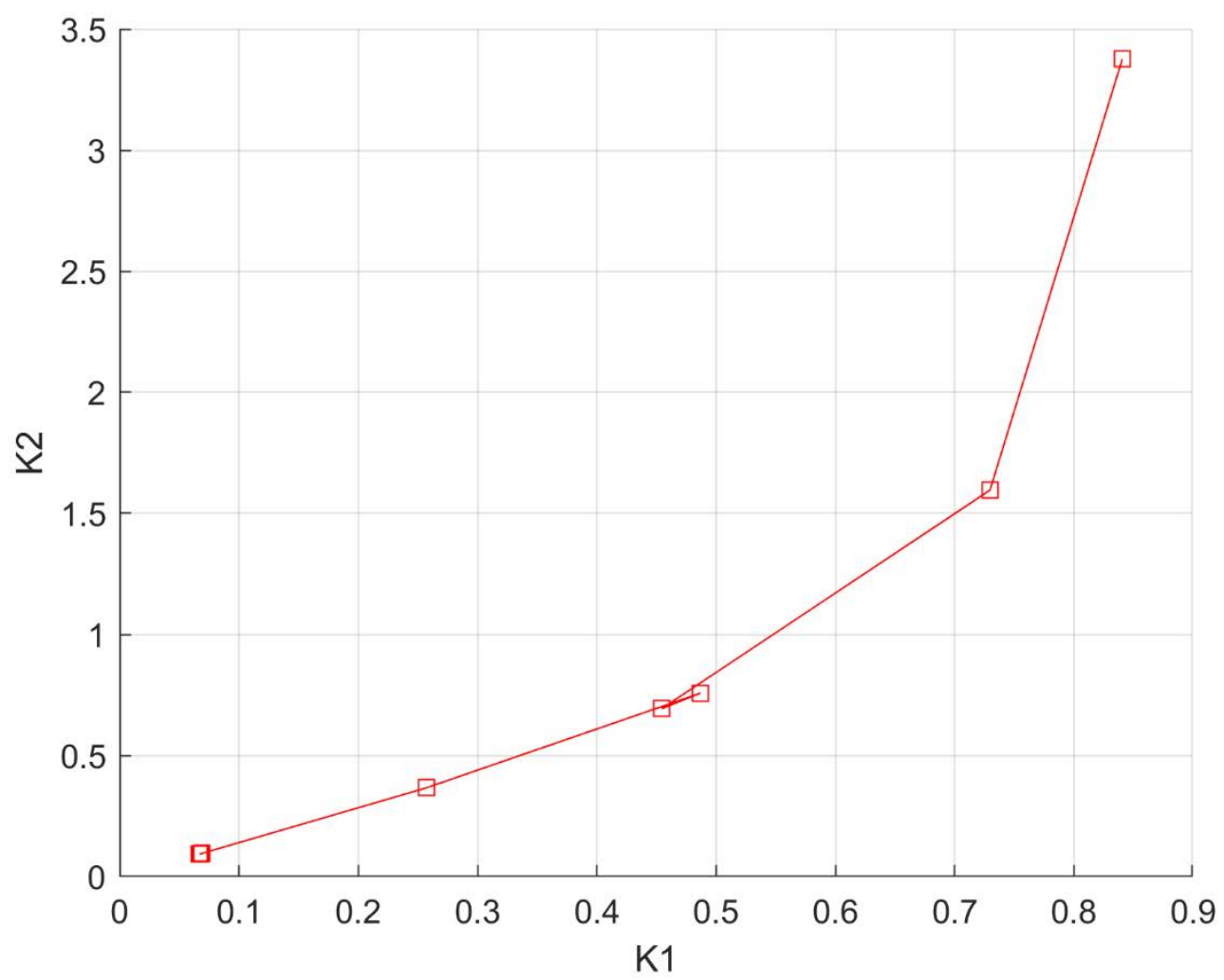

Figure 2. Pareto-optimal set for solving a problem by a method using a genetic algorithm.

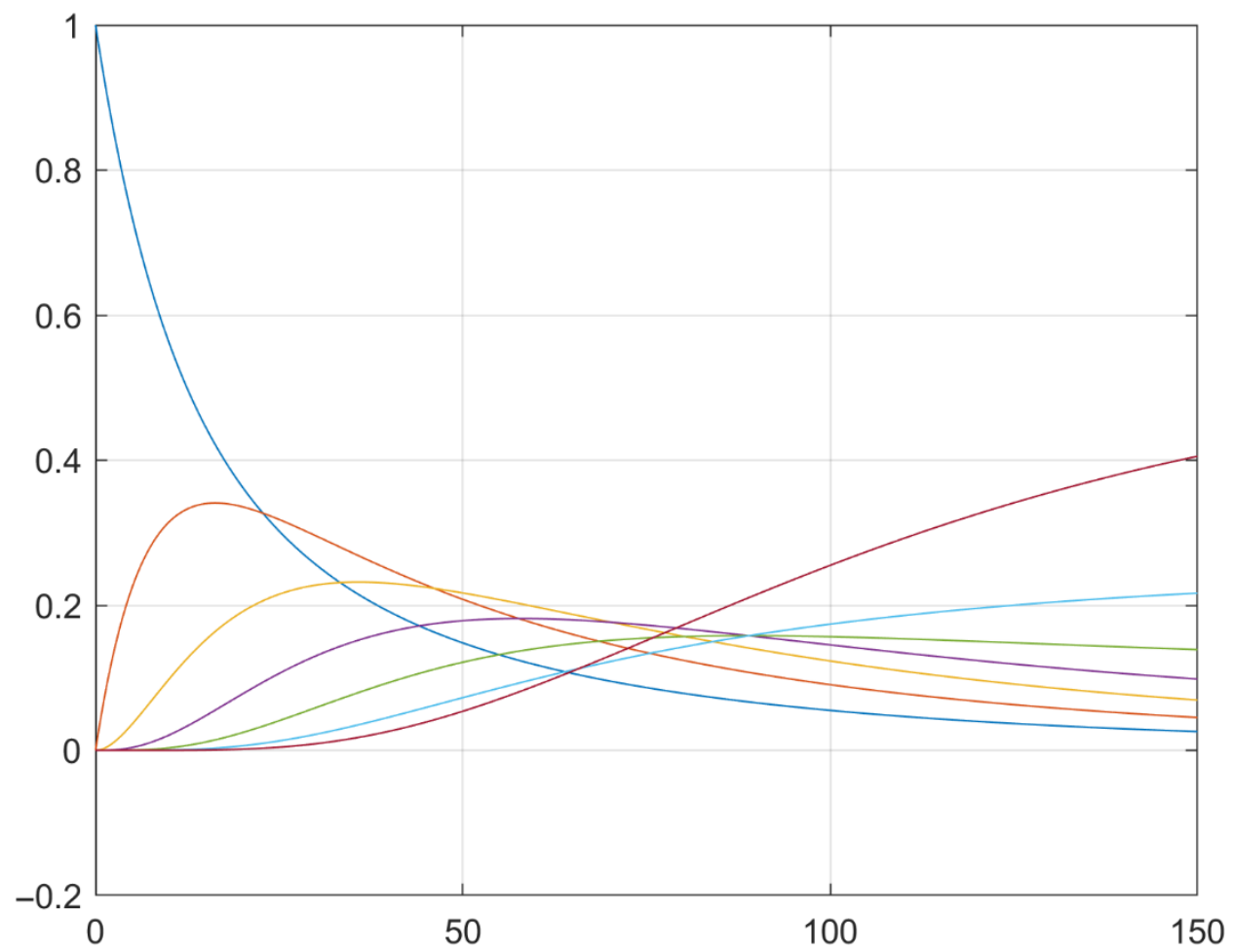

Figure 3. Probability distribution of the system being in a certain state ( $X$-axis is the distribution time, $Y$-axis is the probability of the system being in a certain state). 
Based on the Pareto-optimal set, the decision-maker can choose the most appropriate solution related to the student's homework time. This choice can be based on the method of analysis of hierarchies, taking into account the principles of pairwise comparison of alternatives. In this problem, the following value is selected from the Pareto set: $\xi=0.9$, $K_{1}=0.7296, K_{2}=1.5957$. With these values, the time to complete homework is $17.43 \mathrm{~min}$. The resulting value is $10.3 \%$ more effective than the current time for homework by students and does not contradict the norms of the study load, regulated by the SanPiN. The obtained solution was achieved while minimizing the time for completing homework, provided that the maximum efficiency from the tasks performed was obtained.

\section{Conclusions}

A student's home study work is a very important component of the learning process and is one of the essential forms of its organization. The success of the study as a whole often depends on how successfully the homework is being prepared. In conditions of intensification of learning, the correct organization of homework is especially important; at the same time, a number of questions arise, the solution of which is connected with the improvement of the educational process and with the protection of the student's health. Practice shows that there are a number of problems in organizing and dispensing students' homework.

The article presents one of the possible ways to solve the problem of optimal distribution of the student's home study work. This problem is solved on the basis of the use of neural network devices, queuing theory, and multicriteria optimization method. A mathematical model and a practical problem of finding the optimal parameter of the system are presented, which is the time of homework by students in one lesson. The educational process is considered by taking into account the minimization of the efforts of schoolchildren, provided that the maximum efficiency of this process is achieved. Since the efficiency of mastering the material is influenced by a number of factors in the work, it is proposed to carry out the selection of homework based on the intellectual analysis of the text of the tasks and to evaluate each of the tasks with a number from 0 to 1 . The process of finding the optimal time for completing homework by students is carried out on the basis of a genetic algorithm. We used the queuing theory to determine the correspondence function in the genetic algorithm. This approach makes it possible to solve problems with a high dimension of the system and represent the optimized parameters as a scalar and a vector, which expands the scope of optimization problems.

The result of the search for the optimal time for completing homework by students is proposed to be presented in the form of a Pareto set. The choice of one of the Pareto points is carried out by applying the principle of pairwise comparison of alternatives by the method of analysis of hierarchies. The results were checked, compared with the parameters of SanPiN "Requirements for the conditions and organization of training in educational institutions", and showed a maximum reduction in the time of homework by students by $10.3 \%$.

In comparison with the known algorithms, the proposed method allows one to obtain better convergence and more accurate values of the Pareto set on the considered optimization problem. Therefore, the proposed algorithm, in combination with a neural network and queuing theory, used to solve multicriteria optimization problems, can provide such results of optimization parameters that lead to an increase in the level of homework by students in the shortest possible time. The practical implementation of this study determines the effectiveness of the application of the developed mathematical apparatus. It does not contradict the norms of the teaching load, regulated by the SanPiN "Requirements for the conditions and organization of training in educational institutions."

Author Contributions: Conceptualization, E.P.D.; methodology, E.P.D. and A.V.O.; software, E.P.D. and A.V.O.; validation, E.P.D., A.V.O., M.V.S. and S.V.S.; formal analysis, E.P.D.; investigation, E.P.D. and A.V.O.; resources, E.P.D. and A.V.O.; data curation, E.P.D. and A.V.O.; writing-original draft preparation, E.P.D., A.V.O., M.V.S. and S.V.S.; writing—review and editing, E.P.D. and A.V.O.; 
visualization, E.P.D. and A.V.O.; supervision, E.P.D. and A.V.O.; project administration, E.P.D.; funding acquisition, this research received no external funding. All authors have read and agreed to the published version of the manuscript.

Funding: This research received no external funding.

Institutional Review Board Statement: Not applicable.

Informed Consent Statement: Not applicable.

Conflicts of Interest: The authors declare no conflict of interest.

\section{References}

1. Sanitary Rules SP 2.4.3648-20. Sanitary and epidemiological requirements for the organization of education and training, recreation and health improvement of children and youth; Approved by the Chief state sanitary doctor Russian Federation dated 28 September 2020 N 28. Available online: https:/ / docs.cntd.ru/document/566085656 (accessed on 1 June 2021).

2. Amjad, M.K.; Butt, S.I.; Kousar, R.; Ahmad, R.; Agha, M.H.; Faping, Z.; Anjum, N.; Asgher, U. Recent Research Trends in Genetic Algorithm Based Flexible Job Shop Scheduling Problems. Math. Probl. Eng. 2018, 2018, 9270802. [CrossRef]

3. Viana, M.S.; Junior, O.M.; Contreras, R.C. A Modified Genetic Algorithm with Local Search Strategies and Multi-Crossover Operator for Job Shop Scheduling Problem. Sensors 2020, 20, 5440. [CrossRef] [PubMed]

4. Rarità, L.; Stamova, I.; Tomasiello, S. Numerical schemes and genetic algorithms for the optimal control of a continuous model of supply chains. Appl. Math. Comput. 2021, 388, 125464. [CrossRef]

5. Hu, Y.; Li, J.; Hong, M.; Liu, M.; Man, Y. Short term electric load forecasting model and its verification for process industrial enterprises based on hybrid GA-PSO-BPNN algorithm-A case study of papermaking process. Energy 2019, 170, $1215-1227$. [CrossRef]

6. Demir, L.; Diamantidis, A.C.; Eliiyi, D.T.; O’Kelly, M.E.J.; Tunali, S. Optimal buffer allocation for serial production lines using heuristic search algorithms: A comparative study. Int. J. Ind. Syst. Eng. 2019, 33, 252-270. [CrossRef]

7. Borisenko, A.; Gorlatch, S. Optimizing a GPU-parallelized ant colony metaheuristic by parameter tuning. Lect. Notes Comput. Sci. 2019, 11657, 151-165.

8. Petrosov, D.A.; Lomazov, V.A.; Petrosova, N.V. Model of an Artificial Neural Network for Solving the Problem of Controlling a Genetic Algorithm Using the Mathematical Apparatus of the Theory of Petri Nets. Appl. Sci. 2021, 11, 3899. [CrossRef]

9. Protopopova, J.; Kulik, S. Educational Intelligent System Using Genetic Algorithm. Procedia Comput. Sci. 2020, 169, 168-172. [CrossRef]

10. Aseev, G.G. Methods of data mining in electronic storages: Genetic algorithms. Radio Electron. Comput. Sci. Manag. 2011, 2, 82-85.

11. Yandybaeva, N.V. Genetic algorithm in the problem of optimizing the university curriculum. Mod. High Technol. 2009, 11, 97-98.

12. Su, B.; Xie, N.; Yang, Y. Hybrid genetic algorithm based on bin packing strategy for the unrelated parallel workgroup scheduling problem. J. Intell. Manuf. 2021, 32, 957-969. [CrossRef]

13. Zhang, H.; Wu, F.; Yang, Z. Hybrid approach for a single-batch-processing machine scheduling problem with a just-in-time objective and consideration of non-identical due dates of jobs. Comput. Oper. Res. 2020, 128, 105194. [CrossRef]

14. Sedak, M.; Rosić, B. Multi-Objective Optimization of Planetary Gearbox with Adaptive Hybrid Particle Swarm Differential Evolution Algorithm. Appl. Sci. 2021, 11, 1107. [CrossRef]

15. Wang, L.; Peng, L.; Wang, S.; Liu, S. Advanced backtracking search optimization algorithm for a new joint replenishment problem under trade credit with grouping constraint. Appl. Soft Comput. 2020, 86, 105953. [CrossRef]

16. Chang, G.W.; Lu, H.J.; Chang, Y.R.; Lee, Y.D. An improved neural network-based approach for short-term wind speed and power forecast. Renew. Energy 2017, 105, 301-311. [CrossRef]

17. Christian, I.; Hüsken, M. Improving the Rprop Learning Algorithm. Second International Symposium on Neural Computation. Available online: https://www.researchgate.net/publication/202255088_Improving_the_Rprop_Learning_Algorithm (accessed on 1 June 2021).

18. Makarov, M.A.; Obidin, V.V.; Osipov, A.V.; Filimonov, A.V. Neural Excel VBA Extension Pack: Certificate of State Registration of Computer Programs. Available online: https://new.fips.ru/registers-doc-view / fips_servlet?DB=EVM\&DocNumber=20196170 73\&TypeFile=html (accessed on 1 June 2021).

19. Dogadina, E.P.; Konoplev, A.N.; Belov, A.A.; Kropotov, Y.A.; Proskuryakov, A.Y. Automated control systems for industrial production. M. Berl. Direct Media 2017, 343.

20. Park, K.; Shin, D.; Chi, S. Variable Chromosome Genetic Algorithm for Structure Learning in Neural Networks to Imitate Human Brain. Appl. Sci. 2019, 9, 3176. [CrossRef] 